\title{
ZUR GESELLSCHAFTLICHEN FUNKTION DER LITERATURWISSENSCHAFT
}

In der letzten Zeit wird häufig die Forderung erhoben, die Literaturwissenschaft müsse sich an den Bedürfnissen der Gesellschaft orientieren. Man verweist dabei gern auf die Massenlektüre, insbesondere bestimmte Arten der Trivialliteratur, und verlangt mit pädagogischem Pathos Erziehung zu emanzipativer Bewußtwerdung des Individuums, zu kritischem Verständnis und geistiger Freiheit. Diese seit Humboldt und Fichte immer wieder vertretenen Forderungen würden sicherlich von allen Universitätsangehörigen zumindest theoretisch unterstützt werden, wenn man nicht den haut goût oder gar den Schwefelgeruch jener linksradikalen Sozialrevolutionäre $\mathrm{zu}$ riechen glaubte, die Literaturwissenschaft entweder radikal in Frage stellen oder zu einem Kampfinstrument gegen die bürgerliche Gesellschaft umfunktionieren wollen. Schon die mittlere Generation der deutschen Universitätsanglisten beobachtet solche Tendenzen mit Horror, hat sie doch selbst noch die Folgen der nazistischen Kultur- und Wissenschaftspolitik miterlebt und muß nun befürchten, daß die eigene Disziplin wiederum in den Dienst politischer Indoktrination gestellt werden soll.

Die sozialrevolutionäre Gruppe junger Literaturwissenschaftler stellt aber nicht die eigentliche Gefahr dar, da ihre Konzeptionen bei den literarisch Interessierten nicht ankommen. Sehr viel gefährlicher, weil scheinbar vernünftig, demokratisch und von aktuellem Interesse ist die modische Forderung, die Literaturwissenschaft dürfe sich nicht mehr an einer kleinen intellektuellen Elite ausrichten, sondern müsse sich mit den literarischen Wünschen, Bedürfnissen und Möglichkeiten der breiten Masse unserer Bevölkerung auseinandersetzen, da sie nur auf diese Weise ihre soziale Relevanz nachweisen könne. 
Typisch für diese Tendenz ist der Aufsatz von E. Späth

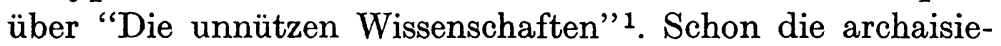
rende Form des Titels soll provokatorisch wirken - und dürfte von den Lesern der altehrwürdigen Anglia auch so aufgefaßt worden sein. Späth ist aber sicherlich kein Umstürzler. Seine besonders "fortschrittlichen" Thesen und Hypothesen werden in den entscheidenden Punkten durch ein "sowohl als auch" oder ein "ja-aber" abgeschwächt. Dennoch bedürfen einige von Späths (keineswegs unnützen) Thesen der Korrektur.

Grundsätzlich wird man die Forderung ablehnen müssen, Literaturwissenschaft habe sich vor allem mit der massenhaft verbreiteten Literatur zu beschäftigen, wenn sie ihre soziale Relevanz erweisen wolle. Nicht um massenhafte Verbreitung geht es primär, sondern um das Problem des Wertes und der Wertung von Texten - gleich nach welchen Kriterien. Späths wesentlichste These (Priorität der Massenliteratur vor den Klassikern) ist literaturtheoretisch nicht fundierbar. Literatursoziologische und leserpsychologische Untersuchungen sind dringend vonnöten. Es ist aber zweifelhaft, ob man sie, noch ehe sie richtig begonnen wurden, mit den Hypotheken der Späthschen Forderung belasten sollte, mit einem ungeklärten Relevanzbegriff und mit einer allein an der Lesehäufigkeit orientierten Auswahl, die implizit das hinreichend bekannte dualistische Wertsystem bisheriger Kitschtheorien ${ }^{2}$

1 E. Späth, "Die unnützen Wissenschaften. Ein anglistischer Beitrag zur Diskussion über die gesellschaftlichen Funktion der Literaturwissenschaften", Anglia, 89 (1971), 199-223.

2 H. Kreuzers Essay ("Trivialliteratur als Forschungsproblem. Zur Kritik des deutschen Trivialromans seit der Aufklärung," $D V j s, 41$ [1967], 173-191) bringt den wohl folgenreichsten Angriff auf diese Theorien. Zu dem Problem allgemein s. J. Schulte-Sasse, Literarische Wertung (Stuttgart, 1971.) und ds., Die Kritite an der Trivialliteratur seit der Aufklärung. Studien zur Geschichte des modernen Kitschbegriffs (München, 1971).

Aus der endlosen Reihe der Vertreter dichotomer Kitschtheorien sollen einige genannt werden: H. Broch, "Einige Bemerkungen zum Problem des Kitsches" und "Das Böse im Wertsystem der Kunst" in ds., Dichten und Erkennen. Essays, vol. I (Zürich, 1955). S. 295350 ; O.F. Bollnow, Das Wesen der Stimmungen (Frankfurt, ${ }^{3} 1956$ ); H.-E. Hass, "Das Problem der literarischen Wertung," Studium Generale, 12 (1959), 727-756; K.M. Michel, "Gefühl als Ware: Zur 
übernimmt: Trivial ist, was viel, hochliterarisch, was kaum und noch dazu von den falschen Leuten gelesen wird.

Der ästhetischen wie der politischen Wertproblematik entzieht sich Späth durch die Behauptung, wissenschaftlicher Beschäftigung wert sei vor allem jene Literatur, die auf ein "weit verbreitetes und echtes Interesse" stößt, d.h., die massenhaft gelesen wird. Milton, Keats, Pope und Shakespeare aber stellen nach Späth keine wichtige geistige Kraft mehr dar: "Man lasse sich von den relativ hohen Auflagenziffern solcher Bücher oder von den großen Besucherzahlen bei Shakespeareaufführungen nicht täuschen. Ein primäres Interesse an den Werken der Klassiker ist heute nicht oft anzutreffen ..." (S. 214). Unterstellen wir einmal die Richtigkeit dieser Behauptung, so ist damit noch nicht bewiesen, daß die Klassiker unwesentlich sind. Ein Autor wie James Joyce war nie populär und wird es nie sein; dennoch hat er - wie andere Genies - ganze Generationen entscheidend beeinflußt. Wir müssen uns darüber klar werden, daß nach Späth der Gegenstand der Literaturwissenschaft durch die bloße Zahl der Leser und damit in nicht wenigen Fällen durch besonders erfolgreiches marketing ("promotion"!) konstituiert wird.

Eher könnte man postulieren, daß die aufgrund kulturspezifischer Übereinstimmung des Wertens seit Jahrhunderten kodifizierten Werke des weltliterarischen Kanons bevorzugt behandelt werden müssen - wenn man schon quantitativ urteilen und begründen will. Außerdem gibt es auch in der mo-

Phänomenologie des Kitsches," Neue Deutsche Hefte, 6 (1959/60), 31-48; L. Giesz, Phänomenologie des Kitsches (Heidelberg, 1960); W. Killy, Deutscher Kitsch. Ein Versuch mit Beispielen (Göttingen, $\left.{ }^{2} 1962\right)$; H. Sauter, "Der Kitsch in der Literatur. Sein Wesen und seine Problematik," Saarbrücker Hefle, 16 (1963), 53-68; J. Elema, "Der Kitsch als Randerscheinung der Kunst," Orbis Litterarum, 21 (1966), 17-38.

Literatursoziologisches Interesse verdient W. Nutz' Arbeit über den Leihbuchroman, Der Trivialroman. Seine Formen und seine Hersteller. Ein Beitrag zur Literatursoziologie (Köln/Opladen, 21965) und die Ergebnisse einer Leserbefragung, durchgeführt durch $\mathrm{H}$. Grimmingers 'Arbeitsgruppe Massenliteratur', "Verwertbare Unmündigkeit. Zur Romanheftserie Jerry Cotton," Ästhetik und Kommunikation, 5/6 (1972), S. 49-57. 
dernen Literatur Äquivalente zu den "Klassikern", die nur von wenigen gelesen werden, aber alle beeinflussen; Beispiele kennt jeder Literaturwissenschaftler. Erst beide Literaturen zusammen ergeben ein Gesamtbild. Man spricht zwar vereinfachend von "Trivialliteratur" und "Hochliteratur", doch handelt es sich dabei nicht (wie die bisher vertretenen dualistischen Theorien lehren) um zwei einander diametral gegenüberstehende Textgruppen, sondern um Extrempositionen auf einer an Mischformen reichen Skala; ihr steht eine ebenso differenzierte Skala von Lesererwartungen, Leserbedürfnissen und Verhaltensweisen gegenüber ${ }^{3}$. Dementsprechend ist auch die Relevanzproblematik nicht durch eine wie immer geartete Setzung zu lösen. Die Literaturwissenschaft nützt niemandem, wenn sie ein Extrem durch ein anderes ersetzt.

Die Bedeutung - wenn nicht der Literaturwissenschaft - so doch der Literatur für das menschliche Leben ist immer wieder festgestellt und bestritten worden. In dieser Beziehung macht das 20. Jahrhundert keine Ausnahme. Es gibt eine große Zahl von Apologeten der Dichtung und der Dichter, und sicherlich gibt es ebenso viele Kritiker, die Literatur und Dichtung für sozial irrelevant halten. Im Zusammenhang dieses Essays braucht nicht die Bedeutung der Literatur allgemein dargestellt zu werden; es kommt nur auf den Nachweis an, daß Literatur und Dichtung spezifische Arten der Interpretation von Welt und Mensch sind und durch andere Arten der Erkenntnis nicht ersetzt werden können. Natürlich spielt auch das Bewußtsein, die Vernunft des Dichters eine große Rolle. Aber erst animus und anima, Verstand und Unbewußtes, sind Grundlage der Imagination und damit der schöpferischen Kraft des Dichters. Daher haben Dichter nach Beendigung ihres Werkes oft kein rationales Bewußtsein ihrer im Werk vermittelten Ideen. Was viele von ihnen zu ihren imaginativen Schöpfungen zu sagen hatten, ist oft im Gegensatz zum Werk weder originell noch erhellend. Goethe, dessen Intelligenz umfassend war, hätte nicht sagen können, was er im ersten Faust zum Ausdruck bringen wollte; erst das Werk hat es ihm gezeigt.

3 Vgl. Kreuzer, passim. 
Der sich durch Imagination vor anderen Menschen auszeichnende Dichter ist auch rationales Wesen. Er wurzelt in einer ganz bestimmten Gesellschaft und wird durch ökonomische Verhältnisse gesteuert. Bestimmte Erlebnisse und Erfahrungen determinieren ihn, so daß er eine Art Spiegel seines Zeitalters ist, wir bestimmte Reflexe der Gesellschaft in seinem Werk wiedererkennen. Aber dichterische Sprache ist nicht nur Darstellung eines nachvollziehbaren rationalen Gehalts, sondern auch zum ganzen Menschen sprechende Kraft, die sich nur des Wörterbuches von animus bedient.

Dichtung und Verstand sind nicht kongruent. Mit Schiller haben zahlreiche Dichter des 20. Jahrhunderts festgestellt, daß die Einbildungskraft die Abstraktion und der kalte Verstand die Dichtung stört. Stellvertretend für viele ähnliche Aussagen stehe die Feststellung R.L. Stevensons, Literatur sei "working far ahead of [...] science, reaching for us [...] effects for which as yet we have no direct name" 4 , Wirkungen, für die es einen exakten wissenschaftlichen Terminus vielleicht nie geben wird.

Die dichterische Sprache bedient sich anderer Ausdrucksmittel und Kommunikationsweisen als die analytische und die philosophische, sie folgt einem eigenständigen, je historisch fixierbaren und damit wandelbaren semantischen Code, den der Dichter der Tradition entnimmt, zuweilen auch umformt, und den das angesprochene Publikum mit dem Autor teilt $^{5}$. Die rationale Analyse des Werks zergliedert seine Einheit und zerstört damit die primäre Rezeption; dennoch müssen sich Deutung und Analyse rationaler Methoden bedienen gegenüber einem Gegenstand, dessen Entstehen und unmittelbare Rezeption rational nicht völlig aufschlüsselbar sind. Versuche, in einem Akt der Einfühlung in den Autor als Quelle der Inspiration und Imagination den Schöpfungsprozeß nachzuvollziehen oder andererseits die subjektiven Eindrücke eines Werks bei je individuellen Rezeptionen als gleichberechtigt und gleich angemessen gelten zu lassen, führen zu nicht

- Familiar Studies of Men and Books (London, 1912; erstmals erschienen 1874 in Cornhill Magazine), S. 8.

5 Vgl. dazu J.M. Lotman, Die Struktur des künstlerischen Textes, edition suhrkamp, Nr. 582 (Frankfurt, 1973), bes. Kap. 8, S. 347 ff. 
weiter generalisierbaren willkürlichen Resultaten ${ }^{6}$. Sie schneiden vor allem die historische Dimension eines jeden Kunstwerks ab, indem sie die eigene Position wissentlich oder unwissentlich verabsolutieren.

Wer "klassische" oder "hohe" Literatur entweder überhaupt nicht oder nur in zweiter Linie als Gegenstand der Literaturwissenschaft akzeptiert, ist folglich in Gefahr, die wesentlichste Dimension der Literatur, ihre spezifische Erkenntnisweise, aus dem Blick zu verlieren. Wer aber die schon von Aristoteles dargestellte typisch poetische Epistemologie ableugnet, wendet sich konsequenterweise auch gegen den literarischen Kanon. Eines der merkwürdigsten Phänomene der Literaturgeschichte ist die Tatsache, daß man sich Jahrhunderte lang wie auf Verabredung darüber einig war, welche Art von Literatur zum Kanon gehört.

Eine der stillschweigend akzeptierten Prämissen läuft darauf hinaus, daß was sich bewährt hat (den "test of time" bestanden hat) gut seine müsse. Aber eine metaphysische absolute Wesenhaftigkeit eines literarischen Werkes und seiner Werte gibt es nicht. Literatur ist nicht nur künstlerisches, sondern auch soziales Phänomen. Selbst Werthaltungen sind Teil des sozialen Verhaltens und daher veränderlich und veränderbar.

Werte sind immer Werte für Menschen. Daher dürfen wir die Normativität oder Apriorität bestimmter Werke der Weltliteratur nicht als kritischer Reflexion enthoben ansehen. Werten begegnen wir immer nur in literarisch vermittelter Form, d.h. Werte werden erst durch zwischenmenschliche Beziehungen faßbar und darstellbar.

Daraus ergibt sich die Folgerung, daß der Literaturwissenschaftler den historischen Kontext, die Bedingungen von Literatur und das gegenseitige Abhängigkeitsverhältnis von Leser und Werk mit untersuchen muß. Die Literatursoziologen

6 Vgl. dazu R. Weimann, Literaturgeschichte und Mythologie. Methodologische und historische Studien (Berlin/Weimar, ${ }^{2} 1972$ ), S. $180 \mathrm{ff}$. und 197. 
haben den berechtigten Vorwurf erhoben, daß wir bisher ohne jede wissenschaftstheoretische Begründung Literatur zur kritischen Behandlung ausgesucht, den Auswahlprozeß aber nicht begründet bzw. analysiert haben?

Das hatte zur Folge, daß nur ein kleiner Teil der Literatur Gegenstand literaturwissenschaftlicher Untersuchungen wurde, und zwar die Werke des sogenannten Kanons, vor allem die Klassiker. Literatur wurde fast ausschließlich unter ästhetischen Gesichtspunkten betrachtet. Die Kriterien selbst sah man als vorgegeben an; es wurde nicht versucht, sie aus bestimmten gesellschaftlichen Verhältnissen abzuleiten oder $\mathrm{zu}$ erklären. Indem Literaturwissenschaft ihren Gegenstand als vorgegeben auffaßte, aber nicht in der Lage war, ihn gegenüber anderen kommunikatorischen Daten abzugrenzen, versagte sie als Wissenschaft.

Jeder Leser - auch der Literaturwissenschaftler - hat scheinbar nur die Möglichkeit zwischen zwei Alternativen: völlige Orientierungslosigkeit außerhalb des primär-erarbeiteten Spektrums oder Übernahme von Beurteilungen aus sekundären Quellen.

Ich habe schon öfters den Verdacht ausgesprochen, daß bestimmte Klassiker noch auf dem Kanon stehen, weil kein Mensch mehr dazu kommt, sie tatsächlich zu lesen. Als Beispiel sei Spensers Fairy Queen genannt. Bewertung und Beurteilung von Literatur sind in einem Maße, das wir uns kaum jemals klar machen, durch die Tradition der Vermittler (Literaturgeschichten, Enzylklopädien, Monographien, Rezensionen etc.) festgelegt. Selbst Büchernarren können nur sehr selektiv lesen; kein Mensch wird jemals aufgrund primärer Lektüre umfassende Kanones kennenlernen.

Um so wichtiger ist es, das Problem des Kanons zum Gegenstand literaturwissenschaftlicher Untersuchungen $\mathrm{zu}$ machen. Wir dürfen in Zukunft dogmatisch gesetzte Kanones nicht mehr ungeprüft als Index nationaler Bildungsgüter bezeichnen. Auch die sogenannte Weltliteratur wird sich im-

"Vgl. dazu die Ưberlegungen von H. R. Jauß, in: "Literaturgeschichte als Provokation der Literaturwissenschaft", in: ds., Literaturgeschichte als Provokation, edition suhrkamp, Nr. 418 (Frankfurt, 1970), S. 144-207. 
mer aufs Neue vor dem kritischen Forum der analytischen Ratio zu rechtfertigen und zu bewähren haben. Vor allem wird man in Zukunft prüfen müssen, wie überhaupt Kanonbildungen zustandekommen. Dabei wird man die kanonkonstituierenden Kriterien auf der Basis historisch bedingter und gewordener Rezeptionen erstellen ${ }^{8}$. Wertfragen und Kriterien der Beurteilung literarischer Werke werden dabei immer wieder im Vordergrund stehen - gleichgültig, ob man sich am "Erwartungshorizont" orientiert, an textimmanenten Unbestimmtheitsstellen, an zeitmorphologischen Kriterien, der epischen Integration, an dem Verhältnis von Aussage und Form.

Nahezu alle bisherigen soziologischen Differenzierungen zwischen Hoch- und Trivialliteratur scheinen mir von falschen Voraussetzungen auszugehen oder an vage benutzten Begriffen zu kranken. Typisch ist z. B. die Unterscheidung hinsichtlich des aktiven, Stellung beziehenden und geistig mitdenkenden Lesers (Hochliteratur) sowie des nur passiv hinnehmenden, seine vom Text ausgelösten Stimmungen genießenden Konsumenten (Trivialliteratur) ${ }^{9}$.

Escarpit ${ }^{10}$ geht davon aus, daß das gebildete Lesepublikum in einen schöpferischen Dialog mit dem Autor eintritt, räumlich also zwischen Buch und Autor steht, während der Konsument anonym bleibt, und ein dialektisches Verhältnis zu Buch und Autor nicht zustande kommt. Damit ist der soziologischen Untersuchung die ästhetische Dichotomie von trivialer und hoher Literatur vorgeschaltet worden, nach der schon seit der Romantik vor allem der Leser für die ästhetische Minderwertigkeit, das "Böse im Wertsystem der

8 Eine Forderung von Jauß, vgl. op. cit., S. $203 \mathrm{ff}$.

- Die Unterscheidung von Gefühl und Stimmung und ibre Anwendung bei der Charakterisierung dieser beiden Lesertypen wurde erstmals von O.F. Bollnow vorgenommen, op. cit., S. $34 \mathrm{f}$., $150 \mathrm{f}$., passim (Erstauflage 1941).

10 R. Escarpit, Das Buch und der Leser (Köln, ${ }^{2} 1966$ ). 
Kunst"11 verantwortlich zu machen ist ${ }^{12}$. Escarpits Resultate fallen entsprechend aus.

Die Crux solcher rigider Dualisierung liegt darin, daß in Ansätzen zutreffend Erkanntes durch Verabsolutierung wieder verfälscht wird und so nuancenreiche Sachverhalte in ein strenges, mit moralischem Impetus vorgetragenes Zweiersystem gepreßt werden. Um bei dem zum Topos erstarrten Beispiel von distanzierter und distanzloser oder Identifikation suchender und Identifikation meidender Rezeption zu bleiben: Es ist durchaus nicht nur Merkmal trivialer Literatur, daß sie ihre Leser das fiktive Element im Leseakt vergessen lassen möchte, und seitens der Leser, daß nur der Trivialliteraturleser sich beim Lesen mit dem Erzählten identifiziere und die dargestellte filstive Welt zumindest eine Zeit lang für wirklich halte. Die gleichen Phänomene gelten bei unterschiedlichen Texten und für unterschiedlich literarisch gebildete Leser ${ }^{13}$. Brochs ${ }^{14}$ Erklärung, darin spiegele sich eine "bestimmte Lebenshaltung", eben die des "Kitsch-Menschen", hilft nicht weiter. Um eine solche Leserreaktion erreichen zu können, muß der Text auf eine beim Leser durch Erziehung und vorangegangene Leseerlebnisse herausgebildete Bereitschaft stoßen. Literarische Bildung und Lektüreerfahrung haben den individuellen Leser auf einen ganz bestimmten Literaturtypus programmiert. Diesem gegenüber ist die psychische Abwehrschwelle gering; andere, vor allem niedere Typen werden dagegen ästhetisch und moralisch verurteilt, ihre Lektüre mit Sanktionen belegt.

1 So der Titel eines Broch'schen Essays über den Kitsch; a.a.O.

${ }_{12} \mathrm{Vgl}$. den Hauptteil von J. Schulte-Sasses Die Kritik an der Trivialliteratur seit der Aufklärung, a.a.O.

${ }^{13}$ Damit soll nicht bestritten werden, daß es einige Werke der Hochliteratur gibt, die - gleichsam theoriekonform - eine Identifikation aufgrund ihrer speziellen Strukturierung ausschließen. $\mathrm{Zu}$ denken wäre etwa an Joyces Ulysses. Die meisten Werke der Hochliteratur legen einer Identifikation keine Hindernisse in den Weg, wenn auch zu untersuchen wäre, welcher Art die von ihnen angezielte Identifikation ist.

14 “Einige Bemerkungen...," S. 295. 
Reflexionen über literarische Texte sind weitgehend textextern bestimmt ${ }^{15}$ : sie richten sich nach dem Bildungsgrad des Lesers, sind abhängig von Sozialisationsprozessen und vom jeweiligen Lesebedürfnis. Literarische Vorlieben sind durch einen Komplex soziologischer, psychologischer und auf Leseerfahrungen beruhender Fakten bestimmt. So ist bei einem literarisch "gebildeten" Leser die Abwehrschwelle gegenüber "literarischen Kunstwerken" besonders gering: Sozialisation und literarisches Training haben ihn entsprechend disponiert. So wie der "gebildete" Leser sich der Hochliteratur psychisch positiv eingestimmt nähert, ist der weniger Gebildete oder Ungebildete einem anderen Literaturtyp gegenüber positiv eingestellt.

Auf das Segment Trivialliteratur schwerpunktmäßig festgelegt, wie Späth vorschlägt, könnten weder Literaturwissenschaft noch Literatursoziologie oder Leserpsychologie umfassende Untersuchungen durchführen. Sie wären aufgrund einer irrationalen, weder ästhetisch noch soziologisch zu rechtfertigenden Entscheidung darauf verwiesen, wahllos das zu untersuchen, was zum Teil zufällig, zum Teil durch Umweltsund gesellschaftliche Einflüsse am meisten gelesen wird. Über die soziale Relevanz der Unterhaltungsliteratur ist gar nicht zu streiten, über die soziale Relevanz einer irrationalen Prinzipien folgenden Literaturwissenschaft aber gewiß.

\section{$* * *$}

Die meisten der bisherigen kitschtheoretischen Ansätze sind davon ausgegangen, wenn dies auch nicht immer so offen gesagt wurde, daß dem Leser von Trivialliteratur gleichsam

15 Das Vorurteil, der Trivialliteraturleser konsumiere gleichsam auf Instinktebene, konnte sich vermutlich deshalb so lange behaupten, weil, da die Lektüre trivialer Texte a priori tabuisiert ist, die ohnehin schon geringer ausgebildete Artikulationsfähigkeit weniger gebildeter Leser so noch verstärkt wird: der gebildete Interviewer scheint derartige Abwehrreaktionen zu verstärken. Dazu und zum Problem der immer noch vorhandenen Tabuisierung trivialer Lektüre s. 'Arbeitsgruppe Massenliteratur', "Verwertbare Unmündigkeit," S. 50. 
von Natur aus bestimmte Eigenschaften des Lesers von Hochliteratur fehlen ${ }^{16}$. Er liest Unterhaltungsliteratur nicht aufgrund eines Mangels, sondern weil er andere Bedürfnisse hat, deren Bedingungen näher zu bestimmen wären, und weil er sehr häufig in anderen Situationen liest; man denke dabei nur an Bus- und Bahnfahrt usw.

Wer als Gymnasiast Fahrschüler war, hat oft eine Literatur kennengelernt, die den ortsansässigen Klassenkameraden unbekannt blieb - nicht umsonst werden bestimmte "Gattungen" unter dem Begriff "Bahnhofskiosk-Literatur" zusammengefaßt. Lektüre dieser Art ist gruppenspezifisch. Es gibt aber Lesemotive, die nach gruppen- bzw. schichtenspezifischen Gesichtspunkten weniger leicht differenziert werden können. Die Love Story oder ähnliche triviale Werke liest auch der Gebildete trotz mangelnder künstlerischer Botschaft aufgrund von Urbedürfnissen, die mit literarischer Bildung nichts zu tun haben. Noch vor wenigen Jahrzehnten führte das Bekenntnis gebildeter Leser zu literarisch anspruchsloser Literatur zu negativen Sanktionen und Prestigeverlust. Das ist heute eher umgekehrt. Es gilt als fashionable, sich zu Trivialliteratur zu bekennen. Belächelt wird, wer sich aus snobistischen Gründen oder aus ehrlicher Überzeugung der Lektüre gängiger Trivialwerke bewußt enthält.

Der Kriminalroman gehört schon seit einem Jahrzehnt zur beliebtesten Lektüre weiter Kreise von Akademikern; unter ihnen befinden sich besonders viele Literaturwissenschaftler. Die Western Novel erhält augenblicklich ihre akademischen Weihen - und zwar nicht nur in der Bundesrepublik. Science Fiction findet ihre fen (pl. von "fan", terminus technicus für Freunde von $S F$ ) sogar unter Naturwissenschaftlern, von denen bekannt ist, daß sie Dichtung und Belletristik allgemein verständnislos gegenüberstehen. Es stimmt also nicht, daß literarisch (oder naturwissenschaftlich) gebildete Leser zur reinen Unterhaltung gelegentlich mit schlechtem Gewissen oder mit mokantem Lächeln in die Niederungen der Literatur hinabsteigen. Eher hat man den Verdacht, daß auf Seiten der

16 Eine solche Deutung legt z.B. Brochs Terminus "Kitsch-Mensch" nahe. 
Leser ein Nivellierungsprozeß vor sich geht, eine Beobachtung, die durch Untersuchungen analoger Fernsehsendungen ( $W e-$ stern, Science Fiction etc.) bestätigt würde.

Aus diesem Grunde müssen wir der bisher stillschweigend als Prämisse unterstellten These, daß der Unterschied zwischen Trivial- und Hochliteratur nicht im Werk, sondern im Leser liege, mit Skepsis gegenübertreten. Hochliteratur, so heißt es, impliziert distanzierten Denkanstoß, Trivialliteratur distanzlosen Konsum. Auf der einen Seite gibt es Leser, die aus Stoffhunger auf sinnlichen Genuß aus sind. Ihnen diametral entgegengesetzt stehen die Asketen, die geistige Anstrengung und Erkenntnis aus gestalteter Form verlangen.

Es wird vorausgesetzt, daß beide Typen von Lesern zusammen nicht kommen können. Der Trivialliteratur-Leser ist geistig träge, reaktionär, auf Konsum und Reproduktion eingestellt; er will passiv unterhalten werden. Der Liebhaber von Hochliteratur dagegen wird als geistig aktiv und interessiert geschildert, politisch progressiv, der Zukunft aufgeschlossen.

Meine Kritik läuft darauf hinaus, daß diese beiden Menschen Typen sind, flat characters, die es in der Wirklichkeit ganz rein nicht bzw. nur selten gibt. Als Darstellung der beiden Endpunkte einer Typenreihe mögen sie nützlich sein. Aber wir müssen der Tatsache eingedenk bleiben, daß im Leben die gemischten Charaktere überwiegen, daß vor allem die $\mathrm{Zu}$ ordnung zu einem der beiden Lager keine Trennung zwischen Schafen und Böcken wie beim Jüngsten Gericht bedeutet. Vielmehr gibt es Wanderer zwischen zwei Welten, Leser von Trivialliteratur aus psychologischen, situativen und mancherlei anderen Gründen. Es ist erwiesen, daß Hochliteratur geistige Anstrengung bedeutet. Da man aber (selbst als Avantgardist) nicht immer unter Hochdruck arbeiten kann, hat Trivialliteratur als Mittel der Entspannung und Unterhaltung eine legitime Funktion.

An welchem Punkt dieses Unterhaltungsbedürfnis in Eskapismus umschlägt und damit in eine asoziale Ersatzbefriedigung, ist bisher noch nicht untersucht worden, da man aus sozial-pädagogischen Gründen Trivialliteratur zunächst einmal (mit Freud) als "milde Narkose" ablehnte. Von Eskapismus würde ich nur sprechen, wenn Lektüre dem Zweck dient, 
einer unbefriedigenden, frustrierenden Umwelt zu entfliehen. A. Franz hat nachgewiesen, daß physisch und psychisch unausgefüllte Personengruppen Ersatzziele suchen und daher in die fiktionale Traumwelt der Trivialliteratur flüchten. So fliehen z.B. Frauen, die sich durch die eintönige Haushaltsarbeit seelisch verarmt fühlen, in die Scheinwelt des Frauenromans. Als weitere wichtige Gruppe führt Franz diejenigen auf, "bei denen das Gefühl ... zu kurz kommt"17, z.B. ältere alleinstehende Menschen, Witwen, Fabrikarbeiterinnen. In all diesen Fällen haben wir nicht den Eskapismus des Lesers zu verdammen, sondern Fehler bzw. Mängel des gesellschaftlichen Systems, mangelndes Gleichgewicht zwischen Mensch und Umwelt, Abbau zwischenmenschlicher Beziehungen, Kontaktschwäche, Technisierung und Industrialisierung, vielleicht auch die conditio humana. Die "Manipulation" des Menschen erfolgt weniger durch die Trivialliteratur als durch Milieu und Gesellschaft; die Trivialliteratur spiegelt sie nur, verstärkt aber vielleicht die zu ihr hinführenden Tendenzen.

Es sollte daher überhaupt keine Frage mehr sein, ob Trivialliteratur wichtig ist - sie ist außerordentlich wichtig, aber aus ganz anderen Gründen als "Hochliteratur". Wie auch immer letztere genannt und definiert wird: sie hat sich seit frühester Zeit dadurch ausgezeichnet, daß sie als kulturell konstitutiv und damit als bedeutsamer und wichtiger als andere Literatur angesehen wurde.

Bedeutende literarische Werke sind in diesem Sinne also solche, die von Zeitgenossen und Nachwelt für bedeutend gehalten wurden. Schon allein durch diese Tatsache waren literarische Werke nicht nur Abbilder ihrer Zeit, sondern formende und beeinflussende Faktoren, die ihrerseits die historische Wirklichkeit mitbestimmten ${ }^{18}$. Sie konnten das um so nachhaltiger, je länger sie für bedeutend gehalten wurden; sie waren weniger bedeutsam, wenn sie als "Eintagsfliegen"

17 A. Franz, "Lesen als Lebensersatz", Bücherei und Bildung, 3 (1951), 673.

18 Vgl. dazu R. Weimann, Literaturgeschichte und Mythologie, bes. das Kapitel "Theater und Gesellschaft in der Shakespeare-Kritik", (Berlin/Weimar, ${ }^{2} 1972$ ), S. $170 \mathrm{ff}$. 
nur kurz Furore machten und dann schnell wieder im literarischen Limbus untertauchten.

$\mathrm{Zu}$ diesen Eintagsfliegen rechne ich nicht Werke eines beliebigen Genres der Trivialliteratur, die als individuelle, einem bestimmten Autor zugehörige Schöpfungen mit distinktiver Physiognomie nur selten in Erscheinung treten: Sie wollen in Aufmachung und Anspruch wie ein Markenartikel konsumiert werden.

Es gibt Beispiele dafür, daß Autorennamen, die beim $\mathrm{Pu}-$ blikum angekommen sind und mit einer bestimmten Art und Qualität von Literatur assoziiert werden, vom Erstautor gegen Honorar an den Verlag ausgeliehen oder an Autoren bzw. -teams verkauft werden. Das individuelle, triviale Werk darf daher nicht einem einzelnen hochliterarischen Werk gegenüber gestellt werden. Die Unterabteilungen der Genres als Vielzahl ähnlicher, nach gleichem Muster verfaßter Werke übernehmen die Rolle der hochliterarisch bedeutsamen Einzelwerke. Ihre Wirkung auf das Lesepublikum ist vorläufig noch eine unbekannte Größe, die durch empirische Forschung analysiert werden muß. In einem rein quantitativ ausgelegten Raster wäre sie wahrscheinlich ähnlich groß wie die der Hochliteratur. Der Beitrag der Bastei-Romane zu Verhaltensweisen, Selbstverständnis und Urteilsfähigkeit heutiger Menschen ist sicherlich ebenso groß wie der Shakespeares, wenn auch Shakespeare einen ganz andersartigen Beitrag zur modernen Kultur geleistet hat: Er ist seit dem 17. Jahrhundert eine lebendige Kraft, die Theater, Drama, Literatur und Kultur entscheidend mitgeprägt hat.

Die Wirkungen der verschiedenen Literaturen müssen also gesondert gewertet werden, aber nicht in der Art, daß man die unerwünschten, von einer bestimmten Zielvorstellung aus negativen Wirkungen ignoriert.

Die jetzige Kultur sähe anders aus, wenn es (um ein Beispiel zu nennen) den Frauenroman nicht gäbe oder gegeben hätte. $\mathrm{Daß}$ von einem solchen Genre nicht gerade sozialrevolutionäre Antriebe ausgehen, wird niemand bestreiten. Man mag diese Literatur auch deswegen schelten, weil sie kapitalistische Gesellschaftsformen fortschreibt und dadurch verfestigt. Ein Blick in die Heftchenliteratur der DDR zeigt aber, daß unter 
ideologischen Vorzeichen ausgerichtete "progressive" Literatur noch weniger ansprechend ist, da die aufklärerische Intention in Agitation und Indoktrination umschlägt. DDRHeftchen verbreiten eine tödliche Langeweile; als Bewohner der BRD neigt man dazu anzunehmen, daß sie nur faute de mieux gelesen werden. Drüben wird offen zugegeben, daß die Heftchenliteratur agitatorischen Zwecken dient. Nach dem Bau des antifaschistischen Schutzwalls, so heißt es, war ein guter Teil der Heftchen nicht mehr erforderlich; zahlreiche Serien stellten ihr Erscheinen ein ${ }^{19}$. Hier bedarf es nicht des Literaturwissenschaftlers, um Manipulation und Indoktrination nachzuweisen; in der Tat gibt es in der DDR keine nennenswerte wissenschaftliche Beschäftigung mit Heftchenliteratur - obwohl die Bedeutung dieses Forschungsgegenstandes des öfteren betont worden ist.

Literatur dieser Art fällt aber auch nicht in den Bereich der von Späth angesprochenen Literatur, da sie nicht auf "echtes Interesse" stößt - ein weiterer Beweis für die Dubiosität des Nachweises sozialer Relevanz von Literatur und Literaturwissenschaft anhand der "Interessen" und "Bedürfnisse" des Publikums. Einige "Interessen" und "Bedürfnisse" können gemacht werden; andere gehören zum Wesen des Menschen und sind daher anthropologische Konstanten. Literaturwissenschaft kann daher nicht von "Interesse" und "Bedürfnissen" als gegebenen Faktoren ausgehen. Für Brecht bestand in den fünfziger Jahren nur seitens einer kleinen Elite ein "echtes Interesse"; mittlerweile wird Brecht in Deutschland häufiger als Shakespeare aufgeführt.

Daraus müssen wir Konsequenzen ziehen für die Arbeit des Literaturwissenschaftlers. Wenn uns die Frage gestellt wird: Ist für den heutigen Leser der zeitgenössische Unterhaltungsroman interessanter als das elisabethanische Drama?, so fällt uns die Antwort nicht schwer. Natürlich ist das elisabethanische Drama interessanter und wichtiger ${ }^{20}$.

19 Edith Gaida, "Belletristische Heftreihenliteratur in der DDR", Weimarer Beiträge, 16, xii (1970).

20 E. Späth, "Die unnützen Wissenschaften", S. 215: "Unterhaltungsroman ist interessanter und damit eben auch wichtiger." 
Der Grund dafür liegt auf der Hand: Das literarische Werk ist Abbild und Agens. Die Dramen der Shakespeare-Zeit haben die soziokulturelle Situation unserer Zeit entscheidend mitgeprägt. Unsere eigene Situation ist daher gar nicht ohne das Studium der elisabethanischen Autoren zu verstehen. Die Lebendigkeit der Werke und auch ihre Bedeutung liegen darin, daß sie zu unserem Wertsystem einen entscheidenden Beitrag geleistet haben.

Erst kraft dieses Beitrags sind wir in der Lage, auch das moderne Drama richtig zu beurteilen, denn die gesellschaftliche Wirklichkeit des modernen Betrachters von Dramen (bzw. generell von Literatur) besteht aus Faktoren, die zum guten Teil geschichtlich vermittelt sind, selbst wenn sie sich gegen die Geschichte wenden. Weimann sagt: "[...] die Spannung zwischen dem Vergangenen und dem Gegenwärtigen ist offensichtlich, und doch sind beide Dimensionen in der Literaturwissenschaft [...] unteilbar. Die Bezogenheit von Geschichtlichkeit und Gegenwart wird in dem Maße fruchtbar, wie das Ursprungs- und das Wirkungsfeld (Shakespeares) konfrontiert werden, also die vergangene Historie kritisch und die gegenwärtige Kritik historisch betrieben werden" ${ }^{21}$.

Anders ausgedrückt: Moderne Literatur ist nicht ohne alte $\mathrm{zu}$ verstehen, ästhetische Wertung und Beurteilung von Literatur ist historisch vermittelt, alte Literatur ist in der neuen lebendig und wirksam. Was wir für gut und richtig halten, beruht auf Denk- und Wertungsprozessen, die am Ende einer langen Auseinandersetzung mit literarischen Grundfragen stehen. Es ist einfach nicht wahr, daß die Literaturwissenschaft ihre gesellschaftliche Relevanz nur schwer beweisen kann. Es hat immer einen literarischen Überbau gegeben und es wird ihn immer geben - selbst wenn in den Schulen einmal keine Literatur mehr gelesen und gelehrt werden sollte. Dieser literarische Überbau wird eine Art Steuermechanismus des kulturellen Bereichs bleiben, selbst wenn, wie Science FictionAutoren befürchten, unsere Zivilisation aufgrund "progressiver Schulsysteme" einer analphabetischen Phase zusteuert ${ }^{22}$.

${ }^{21}$ Robert Weimann, op. cit., S. 25.

${ }^{22}$ H. Beam Piper, Null ABC (Frankfurt/M., 1972), S. 138. 
Damit soll keineswegs die These vertreten werden, an der Literaturwissenschaft, so wie sie heute betrieben wird, dürfe isich nichts ändern. Die Auffassung von Literaturwissenschaft hat sich faktisch insofern bereits gewandelt, als heute ganz allgemein der Literaturbegriff weiter gefaßt wird als vor zwanzig Jahren. Etwa seit Northrop Frye gehören zur Literatur nicht nur die im eigentlichen Sinne "dichterischen" Werke (in Vers und Prosa), sondern Texte jedweder Art.

Ob man mit Frye der Meinung ist, daß Metaphern und Mythen die Sprache der expositorischen Texte ebenso determinieren wie die Literatur, ist dabei von untergeordneter Bedeutung. Unbestritten ist jedenfalls, daß sich Literaturwissenschaft auch mit nicht-literarischen Texten zu beschäftigen hat - und zwar nicht nur, um spezifische Merkmale der dichterischen Sprache zu analysieren. Diese neue Grundlagenwissenschaft, Textlinguistik genannt, gewinnt in neuester Zeit immer größere Bedeutung für die wissenschaftstheoretische Grundlegung des Faches sowie die Operationalisierung und Objektivierung literarischer Texte.

Aber wenn Texte zentraler Gegenstand der Literaturwissenschaften sein werden, so bedeutet das nicht, daß diese Texte nur als autonome Größen wissenschaftlich untersucht werden. Die den Text bedingenden Faktoren müssen vielmehr ebenfalls analysiert werden: die Psyche des Autors, gesellschaftliche und politische Verhältnisse, kulturgeschichtliche Hintergründe, literarische und literaturkritische Traditionen. Als wesentlichster Faktor aber sollte das eigentlich innovatorischkreative Element untersucht werden, da es (oder obwohl es) nicht in demselben Maße Funktion gesellschaftlicher Gegebenheiten ist, sondern der Imagination des Dichters, und damit einer spezifischen seelischen Strukturierung entspringt.

$\mathrm{Zu}$ den Entstehungsbedingungen zählen ferner seelischgeistige Dispositionen des Lesepublikums. Jauß prägte den Begriff des "Erwartungshorizontes" ${ }^{23}$; er besagt, daß jedes literarische Werk für ein bestimmtes Publikum geschrieben wird, das durch einen spezifischen soziokulturellen Kontext determiniert ist. Besonders deutlich wird dieser Faktor bei der

23 "Literaturgeschichte als Provokation", op. cit., passim. 
Produktion von Gebrauchsliteratur, die oft von Autorenteams aufgrund von Leserbefragungen geschrieben wird und somit die literatursoziologische These der sog. "reflecting theory" ${ }^{24}$ bestätigt, jedoch in einer entscheidenden Nuancierung: Nicht die faktischen Gegebenheiten des Lebens werden widergespiegelt, sondern die Wert- und Wunschvorstellungen sowie die Ideale der Leser.

Zur Untersuchung der Entstehungsbedingungen muß komplementär die Erforschung der Rezeption von Literatur treten $^{25}$. Das darf nicht vulgärmarxistisch geschehen, indem das Werk aus einer bestimmten Umwelt und einer bestimmten Publikumsbindung abgeleitet wird; Konfrontation mit einem statischen Gesellschaftsfragment und damit passive Widerspiegelung reichen als Erklärung nicht aus. Erforderlich ist vielmehr die Einbettung in den komplexen Prozeß einer geschichtlichen Bewegung, die nur dialektisch erfaßbar ist.

Bei der Analyse der Rezeptionsbedingungen wird man bald feststellen, daß Rezeptionsakte außerordentlich komplex und schwer kategorisierbar sind. Bewußte oder unbewußte politische und weltanschauliche Haltungen und Dispositionen spielen eine Rolle; Interessen, Stimmungen, literarische Normen und Traditionen, Ergebnisse früher Sozialisation etc.

Eine Literaturgeschichte des Lesers macht deshalb große Schwierigkeiten, weil sie mit ausschließlich literaturwissenschaftlichen Methoden nicht geschrieben werden kann. Daher

24 Ruth A.Inglis, "Das Verhältnis von Literatur und Gesellschaft in objektiver Betrachtung”, in Wege der Literatursoziologie, ed. H. N. Fügen (Neuwied-Berlin, 1968), S. 163-176, hier bes. S. $171 \mathrm{ff}$. Die "control theory" und die "reflecting theory" besagen in ihrem ursprünglichen, von Inglis als Arbeitshypothese aufgestellten Sinn, da B Literatur entweder die Faktizität des gesellschaftlichen Lebens spiegelt (reflecting theory) oder in einem prognostischen Vorgriff gesellschaftliche Zustände darstellt, die sie selbst mit herbeizuführen hilft (control theory). Beide Theorien ließen sich an den von ihr untersuchten Texten nicht belegen und mußten revidiert werden.

${ }^{25}$ Die Bedingungen und Fragestellungen derartiger Forschungen finden sich ausführlich dargestellt in G. Wienold, Semiotik der Literatur, a.a.O. Die theoretisierenden Überlegungen Wienolds müssen allerdings erst noch - was sich als außerordentlich schwierig erweisen dürfte - in praktikable Verfahrensweisen umgesetzt werden. 
rät Erwin Wolff ${ }^{26}$ zur Beschränkung auf den "intendierten Leser", der aus dem Text erschlossen werden kann.

Hier ist die weit verbreitete Furcht erkennbar, daß die Verlagerung des Erkenntnisinteresses auf die Wirkung von $\mathrm{Li}$ teratur den spezifischen Charakter der Literaturwissenschaft unterhöhlen und schließlich zerstören könnte. Aber es führt kein Weg zurück zu einer autonomen Dichtungswissenschaft. Die "methodologische Malaise" (Oppel) unserer Disziplin besteht darin, daß wesentliche Forschungsbereiche an Nachbardisziplinen abgegeben wurden und ein einigendes Zentrum der diversen Forschungsrichtungen verlorengegangen ist.

Die Verselbständigung der Nachbar- und Hilfsdisziplinen beweist nur den Mangel an Zusammenschau und Synthese. Die Literaturwissenschaft muß die Nachbarwissenschaften unter ihrem spezifischen Forschungsgesichtspunkt integrieren und nützen. Nur auf diesem Wege ist für den Literaturwissenschaftler in Zulkunft Forschung möglich.

Gegenstand der Literaturwissenschaft bleibt auch weiterhin der Text und seine Vermittlung; einigendes Zentrum der Literaturwissenschaft ist damit der Bereich zwischen dem literarischen Text und der gesellschaftlichen Wirklichkeit:

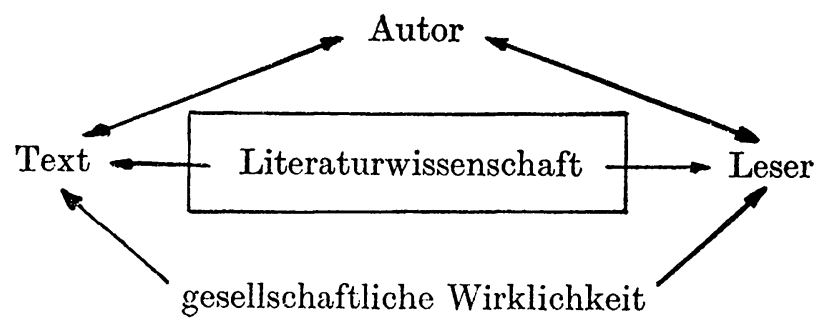

Literaturwissenschaft muß daher Ansätze und Fragestellungen anderer Wissenschaften - der Psychologie, Soziologie, Linguistik und der Kommunikationsforschung - mit einbeziehen. Beim gegenwärtigen Stand der interdisziplinären $\mathrm{Zu}$ sammenarbeit ist nicht $\mathrm{zu}$ hoffen, daß Wissenschaftler dieser

26 "Der intendierte Leser: Ǔberlegungen und Beispiele zur Einführung eines literaturwissenschaftlichen Begriffs", Poetica, 4 (1971), 141-166. 
Fächer sich Probleme der Literaturwissenschaft zu eigen machen; es fragt sich aber auch, ob ein solches Abschieben wichtiger Aufgaben legitim wäre. Untersuchungen über das Leseverhalten $\mathrm{z}$. B. sind für die Literaturwissenschaft nützlich und gehören daher auch in ihr Arbeitsgebiet ${ }^{27}$.

Wir müssen uns dagegen verwahren, daß die soziale Relevanz von Literatur ausschließlich nach der Zahl der verkauften oder gelesenen Exemplare eines Buches berechnet wird. Es sollte mittlerweile klar geworden sein, daß der Literaturwissenschaftler seine Existenzberechtigung auf diese Weise nicht nachweisen kann. Universitäre Forschung innerhalb der neueren Philologien unter dem Gesichtspunkt einer falsch verstandenen Relevanz schwerpunktmäßig auf Konsumliteratur zu verlagern, würde eine Verödung der Disziplin zur Folge haben.

Genauso verfehlt wäre die Ausrichtung der universitären Literaturwissenschaft an den Bedürfnissen der Schule. Natürlich bilden wir an der Universität vor allem zukünftige Lehrer aus - daher muß die Literaturdidaktik stärker betont werden als bisher. Aber daraus darf nicht gefolgert werden, daß die Curricula der Universität sich gegenständlich an den Stoffplänen der Schule zu orientieren haben.

Aufgabe der Literaturwissenschaft ist in Zukunft, das Ursprungs- und Wirkungsfeld von Literatur, die Spannung zwischen Vergangenem und Gegenwärtigem, und damit das gültige Wertsystem genetisch-historisch zu erfassen, zu verstehen, um es (wenn nötig) zu verändern.

Regensburg

Karl Heinz GöLLer

27 Vgl. Späth, S. 217. 